\title{
Índices de oxigenación en los pacientes neuroquirúrgicos utilizando dos estrategias ventilatorias en el perioperatorio
}

\author{
Index of oxygenation in neurosurgical patients using two ventilatory strategies on the perioperatory \\ Índices de oxigenação em pacientes neurocirúrgicos utilizando duas estratégias ventilatórias no perioperatório
}

Eduardo Alberto González Escudero, ${ }^{\star}$ Jesús Salvador Sánchez Díaz, ${ }^{*}$ Ivonn Monserrat Hernández Leyva, ${ }^{*}$ Karla Gabriela Peniche Moguel, ${ }^{\star}$ Josué Eli Villegas Domínguez, ${ }^{\ddagger}$ María Verónica Calyeca Sánchez ${ }^{\star}$

\section{RESUMEN}

Introducción: La comorbilidad pulmonar postoperatoria dependerá de factores relacionados con el paciente, modificables y no modificables, pero definitivamente los parámetros ventilatorios intraoperatorios tienen un rol fundamental. Existen índices de oxigenación (IO) que pueden resultar útiles en la evaluación del grado de lesión pulmonar.

Material y métodos: Estudio prospectivo, descriptivo, analítico, de casos y controles. Pacientes que requirieron intervención neuroquirúrgica (urgente o electiva) y ventilación mecánica invasiva para el procedimiento. Durante el periodo comprendido entre el $1^{\circ}$ de enero de 2018 al 31 de diciembre de 2018. Los pacientes que cumplieron con los criterios de inclusión se asignaron uno a uno para pertenecer al grupo $\mathrm{Vt}$ Intermedio ( $>8 \mathrm{a}<10 \mathrm{~mL} / \mathrm{kg}$ peso predicho) 0 al grupo Vt Bajo (6-8 mL/kg peso predicho). La PEEP fue determinada a consideración del médico (anestesiólogo).

Resultados: En el periodo considerado se incluyeron 60 pacientes, los cuales cumplieron con los criterios de inclusión. Del total, 30 pacientes se incluyeron en el grupo Vt Intermedio (VtI) y 30 pacientes en el grupo Vt Bajo (VtB). La modalidad ventilatoria más utilizada fue asisto-control-volumen (ACV) con $96.7 \%$ para Vtl y $100 \%$ para $\mathrm{VtB}$ con $p=0.3$. El Vt por peso predicho en el grupo de $\mathrm{Vt}$ tuvo una media de $8.9 \mathrm{~mL} / \mathrm{kg}$ y en el grupo de VtB una media de $7.1 \mathrm{~mL} / \mathrm{kg}$ con una $p=0.001$. La escala de coma de Glasgow (ECG) posterior a la extubación fue de 14.3 puntos y 14.4 puntos para Vtl y VtB sin diferencia estadísticamente significativa.

Conclusión: Utilizar volumen corriente intermedio $(>8 \mathrm{a}<10 \mathrm{~mL} / \mathrm{kg}$ peso predicho) en los pacientes neuroquirúrgicos ocasiona alteración de los índices de oxigenación: $\mathrm{PaO}_{2} / \mathrm{FiO}_{2}$ y $\mathrm{PaO}_{2} / \mathrm{PAO}_{2}$. El nivel de PEEP durante el perioperatorio de los pacientes neuroquirúrgicos no ocasiona diferencia significativa en la escala de coma de Glasgow.

Palabras clave: Índices de oxigenación, volumen corriente bajo, volumen corriente intermedio, PEEP, escala de coma de Glasgow.

\section{ABSTRACT}

Introduction: Postoperative pulmonary comorbidity will depend on factors related to the patient, modifiable and non-modifiable, but intraoperative ventilatory parameters definitely play a fundamental role. There are oxygenation indices $(\mathrm{Ol})$ that may be useful in assessing the degree of lung injury.

Material and methods: Prospective, descriptive, analytical, case-control study. Patients who required neurosurgical intervention (urgent or elective) and invasive mechanical ventilation for the procedure. During the period from January 1, 2018 to December 31, 2018. Patients who met the inclusion criteria were assigned 1 to 1 to belong to the Intermediate Tidal Volumen group (ItV) (> 8 to $<10 \mathrm{~mL} / \mathrm{kg}$ predicted weight) or to the Low Tidal Volumen group (LtV) $(6-8$ $\mathrm{mL} / \mathrm{kg}$ predicted weight). PEEP was determined for the doctor's consideration (anesthesiologist).

Results: In the period considered, 60 patients were included who met the inclusion criteria. Of the total, 30 patients were included in the It $V$ group and 30 patients in the LtV group. The most commonly used ventilatory modality was

*Hospital de Especialidades No. 14, Centro Médico Nacional (CMN) «Adolfo Ruiz Cortines", IMSS Veracruz, Veracruz.

‡ Universidad del Valle de México. Facultad de Medicina, Campus Veracruz, México.

Recepción: 04/08/2019. Aceptación: 27/09/2019.

Citar como: González EEA, Sánchez DJS, Hernández LIM, Peniche MKG, Villegas DJE, Calyeca SMV. Índices de oxigenación en los pacientes neuroquirúrgicos utilizando dos estrategias ventilatorias en el perioperatorio. Med Crit. 2020;34(5):265-272. https:// dx.doi.org/10.35366/96456

www.medigraphic.com/medicinacritica asysto-control-volume (ACV) with $96.7 \%$ for ItV and $100 \%$ for LtV with $p=0.3$. The predicted weight $t V$ in the ItV group had an average of $8.9 \mathrm{~mL} / \mathrm{kg}$ and in the $L t V$ group an average of $7.1 \mathrm{~mL} / \mathrm{kg}$ with a $p=0.001$ The Glasgow coma scale (GCE) after extubation was of 14.3 points and 14.4 points for ItV and LtV without statistically significant difference.

Conclusion: Using intermediate tidal volume ( $>8$ to $<10 \mathrm{~mL} / \mathrm{kg}$ predicted weight) in neurosurgical patients, causes alteration of oxygenation rates: $\mathrm{PaO}_{2}$ $\mathrm{FiO}_{2}$ and $\mathrm{PaO}_{2} / \mathrm{PAO}_{2}$. The level of PEEP during the perioperative period of neurosurgical patients does not cause a significant difference in the Glasgow coma scale.

Keywords: Oxygenation rates, low tidal volume, intermediate tidal volume, PEEP, Glasgow coma scale.

\section{RESUMO}

Introdução: A comorbidade pulmonar pós-operatória vai depender de fatores relacionados ao paciente, modificáveis e não modificáveis, mas os parâmetros ventilatórios intra-operatórios certamente têm papel fundamental. Existem índices de oxigenação (IO) que podem ser úteis na avaliação do grau de lesão pulmonar.

Material e métodos: Estudo prospectivo, descritivo, analítico, caso-controle. Pacientes que necessitaram de intervenção neurocirúrgica (urgente ou eletiva) e ventilação mecânica invasiva para o procedimento. Durante o período de $1^{\circ}$ de janeiro de 2018 a 31 de dezembro de 2018. Os pacientes que preencheram os critérios de inclusão foram designados de 1 a 1 para pertencer ao grupo Vt Intermediário (> 8 a $<10 \mathrm{~mL} / \mathrm{kg}$ de peso previsto) ou para o grupo Vt Baixo (6-8 $\mathrm{mL} / \mathrm{kg}$ de peso previsto). A PEEP foi determinada por consideração do médico (anestesiologista).

Resultados: No período considerado, foram incluídos 60 pacientes que atenderam aos critérios de inclusão. Do total, 30 pacientes foram incluídos no grupo Vt Intermediário (VtI) e 30 pacientes no grupo Vt Baixo (VtB). A modalidade ventilatória mais utilizada foi o volume assistido-controlado (VAC) com $96.7 \%$ para $\mathrm{Vtl}$ e $100 \%$ para VtB com $p=0.3$. O Vt previsto em peso no grupo Vtl teve média de $8.9 \mathrm{~mL} / \mathrm{kg}$ e no grupo $\mathrm{VtB}$ média de $7.1 \mathrm{~mL} / \mathrm{kg}$ com $p=0.001$. A escala de coma de Glasgow (ECG) após a extubação foi de 14.3 pontos e 14.4 pontos para Vtl e VtB sem diferença estatisticamente significativa.

Conclusão: $O$ uso de volume corrente intermediário ( $>8$ a $<10 \mathrm{~mL} / \mathrm{kg}$ de peso previsto) em pacientes neurocirúrgicos causa alteração nos índices de oxigenação: $\mathrm{PaO} / \mathrm{FiO}_{2}$ e $\mathrm{PaO}_{2} / \mathrm{PAO}_{2}$. O nível de $\mathrm{PEEP}$ durante o período perioperatório de pacientes neurocirúrgicos não causa diferença significativa na escala de coma de Glasgow.

Palavras-chave: Índices de oxigenação, volume corrente baixo, volume corrente intermediário, PEEP, escala de coma de Glasgow.

\section{INTRODUCCIÓN}

La perspectiva actual de la ventilación mecánica (VM) comenzó en el siglo XVIII con el descubrimiento del oxígeno $\left(\mathrm{O}_{2}\right) ;{ }^{1}$ sin embargo, la poliomielitis marcó la pauta en su progreso. A mediados del siglo XX el Dr. Bjorn Ibsen hizo la primera demostración de la ventilación con presión positiva (VPP), resultando exitosa. Este anestesiólogo danés fue la causa del desarrollo de un gran número de ventiladores regulados por volumen. Para la década de los 60 la VPP fue esencial en 
el apoyo ventilatorio durante la anestesia de cirugías torácicas, ayudando con problemas como las atelectasias del postoperatorio; a posteriori su uso se incorporó en las unidades de cuidados intensivos ( $\mathrm{UCl})$. A finales de los años 80 el conocimiento de funciones clave conduce a la aproximación de aceptar mayor presión parcial de dióxido de carbono (hipercapnia permisiva) como consecuencia necesaria del uso de volumen corriente $(\mathrm{Vt})$ bajo. ${ }^{2,3}$ La tecnología ha respaldado al conocimiento emergente relacionado con el daño iatrogénico que ocurre a nivel pulmonar, consecuencia de la VM, la cual nos brinda la oportunidad de otorgar soporte vital a los pacientes sometidos a procedimientos quirúrgicos mayores o aquéllos críticamente enfermos. ${ }^{4}$ Lo anterior resalta el papel dual de la VM, por un lado, salvar la vida del enfermo y por el otro, complicarla con lesión pulmonar inducida por la ventilación (VILI), concepto descrito en la década de 1970 para definir estos efectos adversos. Por otra parte, la lesión pulmonar asociada a la ventilación (VALI), la cual alude a la exacerbación de una lesión pulmonar preexistente debido a factores relacionados con la VM, mientas que VILI hace referencia a los pulmones previamente sanos. ${ }^{5-7}$ Alrededor de 230 millones de cirugías mayores se realizan cada año en el mundo y la VM es esencial para la anestesia general. Se estima que aproximadamente $20 \%$ de los pacientes que recibe VM en estos procedimientos desarrollará VILI. La comorbilidad pulmonar postoperatoria dependerá de factores relacionados con el paciente, modificables y no modificables, pero definitivamente los parámetros ventilatorios intraoperatorios tienen un rol fundamental. ${ }^{8-10}$ Relacionado a lo anterior, existen dos causas potenciales: sobredistensión alveolar (asociada a Vt) y cizallamiento alveolar asociado al PEEP (Positive End Expiratory Pressure). ${ }^{11}$ En pulmones no lesionados, zonas colapsadas, pero reclutables y zonas sobredistendidas coexisten durante la anestesia general. Dichas zonas tienen expansión no homogénea, siendo más vulnerables a VILI. La cirugía per se, el sangrado, la isquemia-reperfusión y periodos de hipotensión provocan liberación de mediadores inflamatorios, los cuales por sí solos pueden ocasionar lesión pulmonar, sumado al estrés de la VM. Durante este proceso también se involucran variables como la edad y peso del paciente, hábito tabáquico y tipo de cirugía. Cuando en el postoperatorio ocurre insuficiencia respiratoria, ésta contribuye de forma importante en la morbilidad y la mortalidad. Hasta $88 \%$ de los problemas pulmonares ocurrirá dentro de las 24 horas posteriores al finalizar la anestesia y la gran mayoría puede prevenirse. ${ }^{12-16}$ Existen índices de oxigenación (IO) que pueden resultar útiles en la evaluación del grado de lesión pulmonar, entre ellos: presión arterial de oxígeno/fracción inspirada de oxígeno $\left(\mathrm{PaO}_{2} / \mathrm{FiO}_{2}\right)$, presión arterial de oxígeno/presión alveolar de oxígeno, $\left(\mathrm{PaO}_{2} / \mathrm{PAO}_{2}\right)$, delta alveolo-arterial de oxígeno $\left(\triangle(A-a) \mathrm{O}_{2}\right)$, índice respiratorio $\left(\triangle(\mathrm{A}-\mathrm{a}) \mathrm{O}_{2} / \mathrm{PaO}_{2}\right)$ y la presión alveolar de oxígeno $\left(\mathrm{PAO}_{2}\right) .{ }^{17}$ Los $\mathrm{IO}$ no sólo son útiles para determinar la severidad del compromiso pulmonar, también se utilizan como criterio pronóstico en problemas específicos (SDRA, síndrome de distrés respiratorio agudo, retiro de la ventilación mecánica) en los pacientes críticamente enfermos. ${ }^{18,19}$

Evitar la hipoxemia y las atelectasias ha sido la justificación para utilizar Vt alto (>10 $\mathrm{mL} / \mathrm{kg}$ peso predicho) durante la anestesia. Por otro lado, la falta de evidencia de que el uso de PEEP ofrezca beneficios en la mortalidad en esta población de pacientes conlleva a que incluso en la actualidad no se utilice ventilación protectora (6-8 $\mathrm{mL} / \mathrm{kg}$ peso predicho) durante el intraoperatorio, siendo frecuente observar la ventilación no protectora (> $10 \mathrm{~mL} / \mathrm{kg}$ peso predicho) con Vt alto y ausencia de PEEP. En los pacientes con problema neurológico es común el uso de VM durante el procedimiento quirúrgico, incluso mantenerla durante el postoperatorio. Mantener un adecuado nivel de $\mathrm{O}_{2}$, $\mathrm{CO}_{2}$ (dióxido de carbono) y limitar los efectos secundarios de la PEEP en la presión intracraneal implica uso de Vt alto, ausencia de PEEP y $\mathrm{FiO}_{2}$ (fracción inspirada de oxígeno) elevada. De tal manera que la VM tiene un papel fundamental en el cuidado del paciente neuroquirúrgico. ${ }^{20,21}$ Por lo anterior, hemos considerado que una estrategia con volumen corriente intermedio ( $>8 \mathrm{a}<10 \mathrm{~mL} / \mathrm{kg}$ peso predicho) en los pacientes neuroquirúrgicos puede ser igual de útil que el volumen corriente bajo para evitar daño pulmonar asociado a la VM documentado a través de los $I O$, ya que el uso de PEEP no modifica valores de $\mathrm{pH}, \mathrm{PaO}_{2}, \mathrm{PaCO}_{2}$, ni se asocia a mala evolución neurológica (menor escala de coma de Glasgow).

\section{Justificación}

La VM en el paciente neuroquirúrgico sigue siendo un tema no resuelto. Es importante saber que uno de cada cinco pacientes que recibe VM es a consecuencia de alguna alteración neurológica, además este tipo de patologías son la principal razón para que ésta se prolongue. El presente estudio nace de la inquietud por brindar mejores condiciones perioperatorias a los pacientes neuroquirúrgicos, relacionadas a la gran cantidad de complicaciones que pueden presentarse asociadas a la VM. Hablar de protección pulmonar resulta en cualquier contexto un reto, a sabiendas de los paradigmas que existen alrededor de este tema, principalmente cuando hablamos del paciente neuroquirúrgico y su relación con la $\mathrm{PaO}_{2}, \mathrm{PaCO}_{2}$ y presión intracraneal probablemente influenciadas por el Vt y la PEEP. 


\section{MATERIAL Y MÉTODOS}

\section{Objetivo general}

1. Identificar alteraciones en los índices de oxigenación $\left[\left(\mathrm{PaO}_{2} / \mathrm{FiO}_{2}\right),\left(\mathrm{PaO}_{2} / \mathrm{PAO}_{2}\right),\left(\Delta(\mathrm{A}-\mathrm{a}) \mathrm{O}_{2}\right)\right.$, $\left(\triangle(\mathrm{A}-\mathrm{a}) \mathrm{O}_{2} / \mathrm{PaO}_{2}\right)$ y $\left.\left(\mathrm{PAO}_{2}\right)\right]$ de los pacientes neuroquirúrgicos utilizando dos estrategias de ventilación pulmonar.

\section{Objetivos específicos}

1. Determinar si las alteraciones de los índices de oxigenación $\left[\left(\mathrm{PaO}_{2} / \mathrm{FiO}_{2}\right),\left(\mathrm{PaO}_{2} / \mathrm{PAO}_{2}\right),\left(\Delta(\mathrm{A}-\mathrm{a}) \mathrm{O}_{2}\right)\right.$,
$\left(\Delta(\mathrm{A}-\mathrm{a}) \mathrm{O}_{2} / \mathrm{PaO}_{2}\right)$ y $\left.\left(\mathrm{PAO}_{2}\right)\right]$ están asociadas con VILI (lesión pulmonar inducida por la ventilación) en los pacientes neuroquirúrgicos.

2. Determinar si el uso de PEEP está asociado a malos resultados neurológicos (menor escala de coma de Glasgow) en los pacientes neuroquirúrgicos.

\section{Metodología}

Se autorizó por el comité de investigación y ética del hospital. No se realizó consentimiento informado por tratarse de un estudio de riesgo mínimo y de no intervención.

Tabla 1: Características generales de la población.

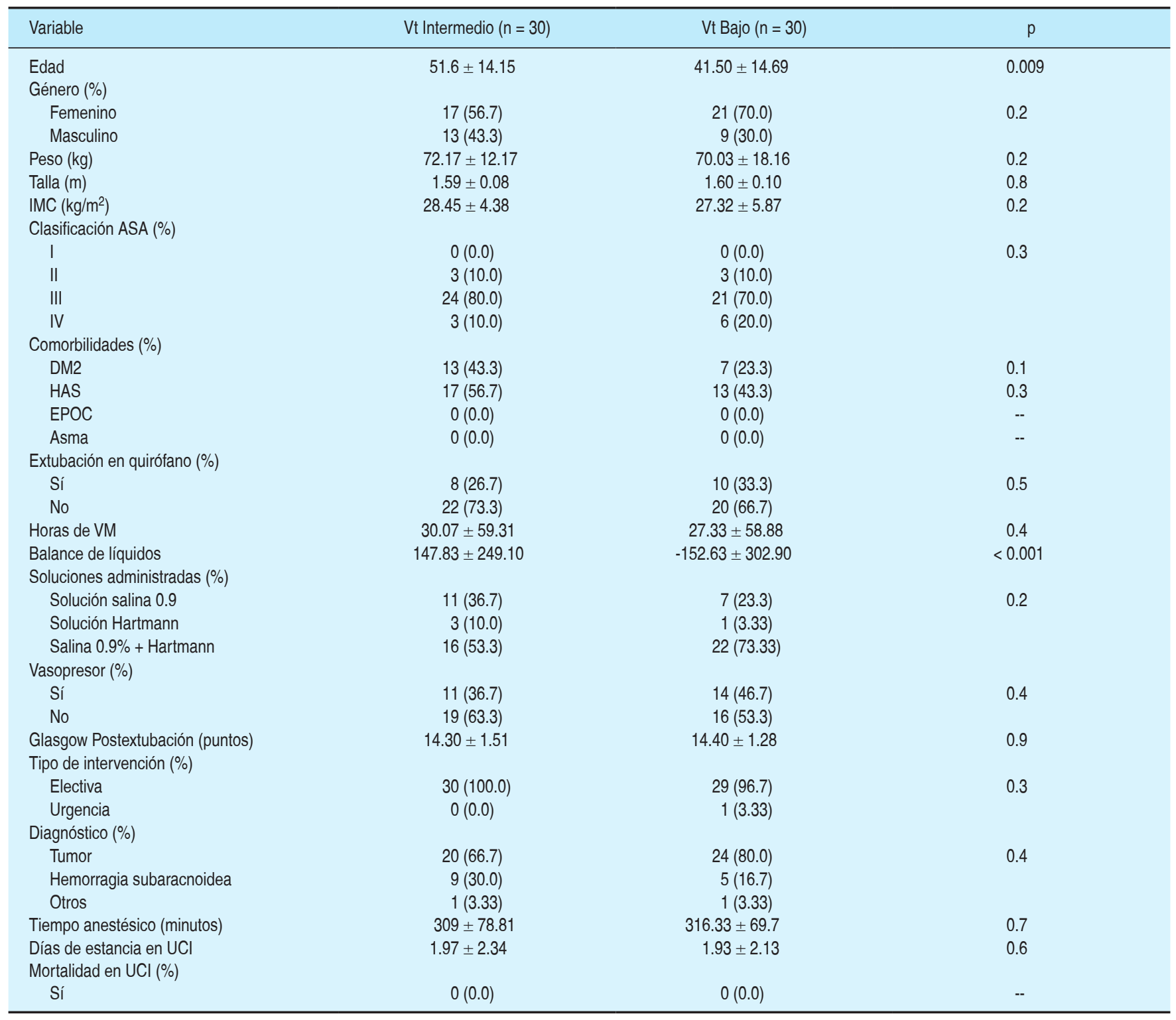

IMC = índice de masa corporal, ASA = American Society of Anesthesiologist, DM2 = diabetes mellitus tipo 2, HAS = hipertensión arterial sistémica, EPOC = enfermedad pulmonar obstructiva crónica, $\mathrm{VM}$ = ventilación mecánica, $\mathrm{UCl}$ = Unidad de Cuidados Intensivos. 


\section{Tipo de estudio}

Estudio prospectivo, descriptivo, analítico, de casos y controles.

\section{Universo de trabajo y lugar de desarrollo}

Pacientes que requirieron intervención neuroquirúrgica (urgente o electiva) y ventilación mecánica invasiva para el procedimiento. Durante el periodo comprendido entre el $1^{\circ}$ de enero de 2018 y el 31 de diciembre de 2018.

\section{Criterios de inclusión}

1. Pacientes mayores de 18 años de edad.

2. Pacientes sometidos a neurocirugía (urgente o electiva).

3. Pacientes con necesidad de ventilación mecánica invasiva para el procedimiento.

4. Pacientes con gasometría arterial posterior a la intubación (cinco minutos) y al finalizar la cirugía.

\section{Criterios de exclusión}

1. Pacientes con registro de variables incompleto.

2. Pacientes que mueran en quirófano.

\section{Criterios de eliminación}

1. Paciente embarazada.

2. Paciente con orden de no reanimación o de máximo alcance terapéutico.

\section{Procedimiento}

Los pacientes que cumplieron con los criterios de inclusión se asignaron uno a uno para pertenecer al grupo Vt Intermedio (> $8 \mathrm{a}<10 \mathrm{~mL} / \mathrm{kg}$ peso predicho) o al grupo Vt Bajo (6-8 $\mathrm{mL} / \mathrm{kg}$ peso predicho). La PEEP fue determinada a consideración del médico (anestesiólogo). Se tomó una gasometría arterial dentro de los cinco minutos posteriores a realizar la intubación y otra al finalizar la cirugía. Se realizó el registro de las variables en la hoja de recolección de datos. Se consideró sobreviviente el paciente al ser dado de alta de $\mathrm{UCl}$ o al llegar al día 30 de estancia. Se utilizó la siguiente fórmula: peso predicho, hombres: $50+0.91$ [altura $(\mathrm{cm})-152.4$ ], mujeres: $45+0.91$ [altura $(\mathrm{cm})-152.4$ ] $\times$ Vt. Se realizó vaciado de datos en hoja de Excel para posteriormente ser analizado con el programa SPPSTM 22.

\section{Análisis estadístico}

Los datos se analizaron mediante estadística descriptiva e inferencial para determinar las características generales de la población. Las variables categóricas se analizaron con $\chi^{2}$ y prueba exacta de Fisher, las variables continuas con prueba de t-Student. Se usó U-Mann-Whitney para variables de distribución anormal. Se utilizó el coeficiente de correlación de Pearson $(r)$, tanto en dirección positiva como negativa. Se consideró significancia estadística con un valor de $p<$ 0.05 . El análisis estadístico se realizó con el programa SPSSTM 22.

\section{RESULTADOS}

En el periodo considerado se incluyeron 60 pacientes, los cuales cumplieron con los criterios inclusión. Del total, 30 pacientes se incluyeron en el grupo Vt Intermedio ( $\mathrm{Vtl}$ ) y 30 pacientes en el grupo $\mathrm{Vt}$ Bajo (VtB). La media de edad en el grupo de Vtl fue 51.6 años, mientras que en el VtB fue 41.5 años con $p=0.009$. El género más frecuente fue el femenino con $56.7 \%$ para $\mathrm{Vtl}$ y $70 \%$ para $\mathrm{VtB}$ con $\mathrm{p}=0.2$. En las variables demográficas como peso, talla e índice de masa corporal (IMC) no existió diferencia estadísticamente significativa. ASA (American Society of Anesthesiologists) III fue lo más frecuente con $80 \%$ para $\mathrm{Vtl}$ y $70 \%$ para VtB con $p=0.3$. La diabetes mellitus tipo 2 (DM2) y la hipertensión arterial sistémica (HAS) fueron las comorbilidades más observadas con $p=0.1$ y $p=0.3$ respectivamente. La mayoría de los pacientes se extubaron en la unidad de cuidados intensivos (UCI) $73.3 \%$ $(\mathrm{Vtl})$ y $66.7 \%(\mathrm{VtB})$ con $\mathrm{p}=0.5$. El tiempo de ventilación mecánica no tuvo diferencia estadísticamente significativa $(p=0.4)$. Al analizar las características de la terapia con líquidos se encontró que a la mayoría de los pacientes se les administraron soluciones cristaloides, solución salina $0.9 \%$ (SS0.9\%) con $36.7 \%$ para $\mathrm{Vtl}$ y $23.3 \%$ para $\mathrm{VtB}$, solución Hartmann ( $\mathrm{SH})$ con $10 \% \mathrm{Vtl}$ y $3.33 \% \mathrm{VtB}$ o una combinación de ambas, Vtl $53.3 \%$ y VtB $73.3 \%$ con $p=0.2$. La cantidad media del balance de líquidos fue $147.83 \mathrm{~mL}$ para $\mathrm{Vtl}$ $y-152.63 \mathrm{~mL}$ para $\mathrm{VtB}$ con $\mathrm{p} \leq 0.001$. La mayoría de los pacientes no necesitó vasopresor con $36.7 \%$ para Vtl y $23.3 \%$ para VtB con $p=0.4$. Las causas diagnósticas por las cuales los pacientes fueron sometidos a neurocirugía fueron: tumor $66.7 \%$ para el grupo de $\mathrm{Vtl}$ y $80 \%$ para el grupo de VtB con $p=0.4$. Con respecto al tipo de intervención quirúrgica (electiva o urgente) no se encontró diferencia estadísticamente significativa. El tiempo anestésico fue de 309 minutos para Vtl y 316.33 minutos para VtB con $p=0.7$. La escala de coma de Glasgow (ECG) posterior a la extubación fue de 14.3 puntos y 14.4 puntos para Vtl y VtB sin diferencia estadísticamente significativa. Los días de estancia en UCl fueron 1.97 días y 1.93 días para Vtl y $\mathrm{VtB}$ respectivamente con $\mathrm{p}=0.6$. La mortalidad fue de $0 \%$ en ambos grupos (Tabla 1). 
Tabla 2: Análisis bivariado de los índices de oxigenación.

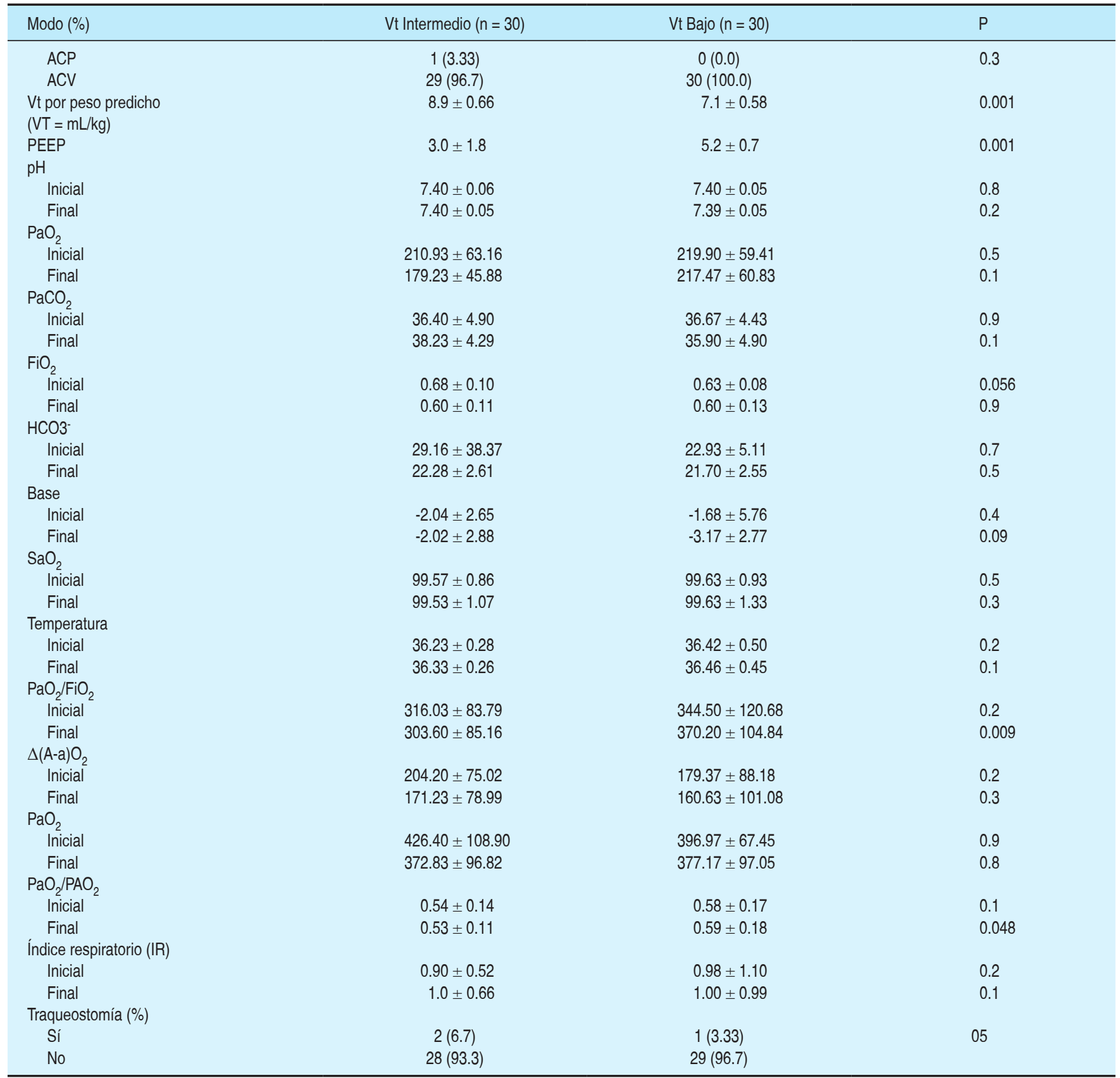

$\mathrm{ACP}=$ asistida/controlada por presión, $\mathrm{ACV}=$ asistida/controlada por volumen, $\mathrm{Vt}=$ volumen corriente, $\mathrm{PEEP}=$ presión positiva al final de la espiración, $\mathrm{pH}=\mathrm{potencial}$ de hidrogeniones, $\mathrm{PaO} 2$ = presión arterial de oxígeno, $\mathrm{PaCO}_{2}=$ Presión arterial de dióxido de carbono, $\mathrm{FiO}_{2}=$ fracción inspirada de oxígeno, $\mathrm{HCO}^{-}=$bicarbonato, $\mathrm{SaO}_{2}=$ saturación arterial de oxígeno, $\mathrm{PaO}_{2} / \mathrm{FiO}_{2}=$ presión arterial de oxígeno/fracción inspirada de oxígeno, $\Delta(\mathrm{A}-\mathrm{a}) \mathrm{O}_{2}=$ diferencia alveolo-arterial de oxígeno, $\mathrm{PaO} \mathrm{O}_{2}=$ presión alveolar de oxígeno, $\mathrm{PaO}_{2} / \mathrm{PAO}_{2}=$ presión arterial de oxígeno y presión alveolar de oxígeno.

En la Tabla 2 observamos que la modalidad ventilatoria más utilizada fue asisto-control-volumen (ACV) con $96.7 \%$ para $\mathrm{Vtl}$ y $100 \%$ para VtB con $\mathrm{p}=0.3$. El Vt por peso predicho en el grupo de $\mathrm{Vtl}$ tuvo una media de $8.9 \mathrm{~mL} / \mathrm{kg}$ y en el grupo de VtB una media de $7.1 \mathrm{~mL} /$ $\mathrm{kg}$ con una $p=0.001$. La PEEP media utilizada fue de $3.0 \mathrm{cmH}_{2} \mathrm{O}$ y de $5.2 \mathrm{cmH}_{2} \mathrm{O}$ para el grupo de Vtl y VtB respectivamente con una $\mathrm{p}=0.001 \mathrm{En}$ las variables $\mathrm{pH}$,
$\mathrm{PaCO}_{2}, \mathrm{HCO} 3-$, Base, $\mathrm{SaO}_{2}, \mathrm{FiO}_{2}$, temperatura final no existió diferencia estadísticamente significativa. De los índices de oxigenación la $\mathrm{PaO}_{2} / \mathrm{FiO}_{2}$ (media Vtl 303.60 $\mathrm{mmHg}$ y VtB $370.20 \mathrm{mmHg}$ ) y la $\mathrm{PaO}_{2} / \mathrm{PAO}_{2}$ (media Vtl 0.53 y $\mathrm{VtB} 0.58$ ) finales presentaron significancia estadística con $p=0.009$ y $p=0.048$ respectivamente. Los demás índices de oxigenación como: $\Delta(\mathrm{A}-\mathrm{a}) \mathrm{O}_{2}, \Delta(\mathrm{A}-\mathrm{a})$ $\mathrm{O}_{2} / \mathrm{PaO}_{2}(\mathrm{IR})$ y $\mathrm{PAO}_{2}$ no tuvieron relevancia estadística. 
Los pacientes del grupo de Vtl requirieron traqueostomía en $6.7 \%$ a diferencia del grupo de $\mathrm{VtB}$ con $3.33 \%$ $(p=0.5)$.

Se realizó correlación de las variables: edad, balance de líquidos, $\mathrm{PEEP}, \mathrm{PaO}_{2} / \mathrm{FiO}_{2}$ y $\mathrm{PaO}_{2} / \mathrm{PAO}_{2}$, las cuales tuvieron diferencia estadísticamente significativa en el análisis bivariado, con la estrategia ventilatoria: Vtl y VtB. Para la edad se obtuvo una $r=-0.356$ con $p=$ 0.005 , balance de líquidos $r=-0.496$ con $p=0.000$, PEEP $r=0.718$ con $p=0.000, \mathrm{PaO}_{2} / \mathrm{FiO}_{2} r=0.350$ con $\mathrm{p}=0.006$ y $\mathrm{PaO}_{2} / \mathrm{PAO}_{2} \mathrm{r}=0.257$ con $\mathrm{p}=0.047$ (Tabla 3). No se encontró correlación entre el nivel de PEEP con la $\mathrm{PaO}_{2} / \mathrm{FiO}_{2}(r=0.153$ con $\mathrm{p}=0.24)$ y la $\mathrm{PaO}_{2} /$ $\mathrm{PAO}_{2}(r=0.064$ con $p=0.62)$. Tampoco existió correlación entre el balance de líquido con la $\mathrm{PaO}_{2} / \mathrm{FiO}_{2}(\mathrm{r}=$ -0.14 con $p=0.26)$ y la $\mathrm{PaO}_{2} / \mathrm{PAO}_{2}(r=0.078$ con $p=$ $0.55)$.

\section{DISCUSIÓN}

Desde 1999 Marco Ranieri y colaboradores ${ }^{22}$ han resaltado el concepto «ventilación mecánica protectora» al comparar dos estrategias ventilatorias en pacientes con SDRA. Por un lado, un grupo con una media de Vt de 11 $\mathrm{mL} / \mathrm{kg}$ peso y por el otro, el grupo control con Vt de 7.6 $\mathrm{mL} / \mathrm{kg}$ peso. La conclusión: utilizar Vt «bajo» disminuye mediadores inflamatorios. Un año después aparece lo que hoy consideramos un texto "clásico y obligatorio" cuando aludimos este tema, ARDS Network ${ }^{23}$ que analiza dos grupos: Vt bajo $(6 \mathrm{~mL} / \mathrm{kg}$ peso predicho y 30 $\mathrm{cmH}_{2} \mathrm{O}$ de presión meseta) y Vt alto $(12 \mathrm{~mL} / \mathrm{kg}$ peso predicho y $50 \mathrm{cmH}_{2} \mathrm{O}$ de presión meseta). Lo anterior resultó en menor mortalidad a 28 días (31 vs. 39.8\%, $p=0.007$ ). Es importante resaltar que el estudio fue detenido por evidente diferencia en la sobrevida. Recientemente, el grupo de investigadores de PReVENT ${ }^{24}$ documentó que utilizar volumen corriente intermedio (8-10 mL/kg peso predicho) en pacientes sin SDRA no incrementa los días de ventilación mecánica, el tiempo de estancia en hospital o en la $\mathrm{UCl}$, no hay diferencia en la mortalidad a 28 o 90 días, tampoco incrementó los eventos adversos (desarrollo de SDRA, neumonía, atelectasias, neumotórax). Pero los efectos nocivos de

Tabla 3: Correlación de variables con la estrategia ventilatoria (Vtl y VtB).

\begin{tabular}{lcc}
\hline Variable & $r$ & $p$ \\
\hline Edad & -0.356 & 0.005 \\
Balance de líquidos & -0.496 & 0.000 \\
$\mathrm{PEEP}$ & 0.718 & 0.000 \\
$\mathrm{PaO}_{2} / \mathrm{FiO}_{2}$ & 0.350 & 0.006 \\
$\mathrm{PaO}_{2} / \mathrm{PaO}_{2}$ & 0.257 & 0.047 \\
\hline
\end{tabular}

PEEP $=$ presión positiva al final de la espiración, $\mathrm{PaO}_{2} / \mathrm{FiO}_{2}=$ presión arterial de oxígeno/fracción inspirada de oxígeno, $\mathrm{PaO}_{2} / \mathrm{PaO}_{2}=$ presión arterial de oxígeno y presión alveolar de oxígeno. utilizar Vt alto se presentan aun cuando ventilamos a los pacientes por periodos cortos, siendo un claro ejemplo de esta situación durante la cirugía. ${ }^{25,26}$ En nuestro trabajo la media de Vt por peso predicho fue de $8.9 \mathrm{~mL} /$ $\mathrm{kg}$ para $\mathrm{Vtl}$ y de $7.1 \mathrm{~mL} / \mathrm{kg}$ para $\mathrm{VtB}(\mathrm{p} \leq 0.001)$. No encontramos diferencia estadísticamente significativa en el tiempo de VM, días de estancia en UCI o en la mortalidad, pero sí en la $\mathrm{PaO}_{2} / \mathrm{FiO}_{2}$ y la $\mathrm{PaO}_{2} / \mathrm{PAO}_{2}(\mathrm{p}$ $=0.009$ y $p=0.048$ respectivamente) correlacionada $\mathrm{a}$ la estrategia ventilatoria (Vtl y VtB). Dichas alteraciones en estos índices de oxigenación podrían reflejar el principio del VILI. Los diferentes estudios realizados hasta hoy han demostrado VILI documentando deterioro respiratorio postoperatorio (insuficiencia respiratoria aguda tipo 1: alteraciones en la ventilación/perfusión, en la difusión, en los cortocircuitos o tipo 2: hipoventilación), atelectasias, neumonía, SDRA, más tiempo de VM, estancia más prolongada en el hospital o incremento de la mortalidad. De manera más fina algunos otros han medido mediadores inflamatorios (interleucinas). ${ }^{27}$ Nosotros decidimos utilizar los índices de oxigenación, los cuales tienen utilidad para evaluar gravedad, causa, tratamiento, evolución y pronóstico de los problemas pulmonares. Sencillamente, el intercambio de oxígeno a este nivel, una solución simple a un problema complejo. ${ }^{28}$

Desde 1974 se introdujo el índice $\mathrm{PaO}_{2} / \mathrm{FiO}_{2}$, aunque se vuelve realmente popular al ser incluido en la definición de SDRA del Consenso Americano-Europeo en 1994. ${ }^{29}$ Depende de la presión barométrica (PB) y de la fracción inspirada de oxígeno $\left(\mathrm{FiO}_{2}\right)$; a nivel del mar, en condiciones de salud, su valor normal sería $>400$ $\mathrm{mmHg}(98 \mathrm{mmHg} / 0.21 \%=466.6 \mathrm{mmHg})$. Sin embargo, el valor «necesario» para iniciar el proceso de weaning es de $150 \mathrm{mmHg}$, otro tópico donde se ha convertido en una variable realmente «importante». La variación del porcentaje de $\mathrm{FiO}_{2}$ utilizada se reflejará en los cortocircuitos medidos y una $\mathrm{FiO}_{2}$ de uno mitigará el efecto de las alteraciones de la ventilación/perfusión. Por lo anterior, la relación $\mathrm{PaO}_{2} / \mathrm{FiO}_{2}$ y la $\mathrm{FiO}_{2}$ no tienen una correlación lineal perfecta. ${ }^{30-32} \mathrm{El}$ índice $\Delta(\mathrm{A}-\mathrm{a}) \mathrm{O}_{2}$ utilizado con frecuencia para evaluar problemas pulmonares tiene la característica de ser altamente sensible, pero poco específico. Aunque útil para discernir entre la hipoxemia por hipoventilación $\left(\Delta(\mathrm{A}-\mathrm{a}) \mathrm{O}_{2}\right.$ normal $)$ de aquella hipoxemia causada por alteraciones en la ventilación/perfusión, difusión o cortocircuitos $\left(\Delta(\mathrm{A}-\mathrm{a}) \mathrm{O}_{2}\right.$ elevado). En condiciones normales influido considerablemente por la $\mathrm{FiO}_{2}$ y en menor medida por la edad. El índice respiratorio $\Delta(\mathrm{A}-\mathrm{a}) \mathrm{O}_{2} / \mathrm{PaO}_{2}$ aparece en 1973. Se ve poco influido por la $\mathrm{FiO}_{2}$ y muy relacionado a la hipoxemia por cortocircuitos, lo que le da utilidad pronóstica en patologías relacionadas (SDRA o neumonía), no así en aquéllas donde predominen las alteraciones de la ventilación/perfusión. ${ }^{32,33} \mathrm{El}$ índice $\mathrm{PaO}_{2} / \mathrm{PAO}_{2}$ se 
ha utilizado desde 1965 con la particularidad de ser el menos afectado por las variaciones de la $\mathrm{FiO}_{2}$, lo que lo convierte en más confiable y específico. Tiene alta capacidad para discriminar la hipoxemia secundaria a cortocircuitos «pura» de la combinada con otros mecanismos. ${ }^{34}$ Como podemos observar la variable más importante al referirnos a los índices de oxigenación es la $\mathrm{FiO}_{2}$, lo que puede ocasionar variación de los resultados. En nuestro estudio la $\mathrm{FiO}_{2}$ inicial para el grupo de Vtl tuvo una media de 0.68 y para el grupo de VtB de $0.63(p=0.056)$, la $\mathrm{FiO}_{2}$ final fue de 0.60 y 0.60 para $\mathrm{Vtl}$ y $\mathrm{VtB}$ respectivamente con $\mathrm{p}=0.9$. Por lo tanto, consideramos que las diferencias que existen entre el grupo de $\mathrm{Vtl}$ y el grupo de VtB en los índices de oxigenación son un reflejo de problemas en el intercambio de oxígeno relacionados con un problema pulmonar, en este caso VILI. Lo anterior se justifica con la correlación de la estrategia ventilatoria (Vtl y VtB) con la $\mathrm{PaO}_{2} / \mathrm{FiO}_{2}(\mathrm{r}$ $=0.35$ con $p=0.006)$ y la $\mathrm{PaO}_{2} / \mathrm{PAO}_{2}(r=0.257$ con $p$ $=0.047)$. Por otro lado, no existió correlación entre la PEEP y el balance de líquidos con la $\mathrm{PaO}_{2} / \mathrm{FiO}_{2}$ y la $\mathrm{PaO}_{2} / \mathrm{PAO}_{2}$.

La autorregulación cerebral (vasodilatación o vasoconstricción) dependerá de factores metabólicos como la $\mathrm{PaCO}_{2}$ principalmente, en menor proporción por la $\mathrm{PaO}_{2}$ y el $\mathrm{pH}$, variables sin relevancia estadística entre ambos grupos de estudio. Dentro de los factores mecánicos encontramos la presión arterial (PA) y a la presión intracraneal (PIC). ${ }^{35}$ Esta última vinculada a la PEEP, la cual incrementa la presión intratorácica pudiendo incrementar la PIC por disminución del retorno venoso cerebral o caída de la presión de perfusión cerebral (PPC). ${ }^{36}$ De esto nace la controversia en cuanto al uso de PEEP en pacientes neurocríticos. Aunque realmente los cambios en la PIC son mínimos y asociados a la disminución de la distensibilidad pulmonar (SDRA, neumonía, edema agudo pulmonar, entre otros). Es incierto el nivel correcto de PEEP en estos pacientes, pero parece que valores de hasta $10 \mathrm{cmH}_{2} \mathrm{O}$ son bien tolerados con distensibilidad pulmonar conservada. ${ }^{37}$ Ningún paciente de nuestra población de estudio tuvo antecedente de problema pulmonar crónico, y ningún paciente tuvo distensibilidad pulmonar disminuida por problema pulmonar agudo. La PEEP utilizada fue de $3.0 \mathrm{cmH}_{2} \mathrm{O}$ para el grupo de Vtl y $5.2 \mathrm{cmH}_{2} \mathrm{O} \mathrm{VtB}$ respectivamente $(\mathrm{p}=$ 0.001), aplicado a juicio del médico (anestesiólogo). La escala de coma de Glasgow (ECG) tuvo una media de 14.3 puntos en el grupo de Vtl y 14.4 puntos en el grupo de $\mathrm{VtB}$ con una $\mathrm{p}=0.9$. Por lo que niveles de $5 \mathrm{cmH}_{2} \mathrm{O}$ de PEEP son bien tolerados en esta población de pacientes sin influir en el resultado neurológico.

Finalmente, uno de los estudios más representativos de este tema fue publicado por Futier y colaboradores en el año 2013. ${ }^{38}$ IMPROVE (Intraoperative Protective Ventilation) incluyó 400 pacientes con cirugía abdomi- nal mayor. Se utilizó en un grupo Vt de 10-12 mL/kg de peso predicho, sin PEEP versus Vt de $6-8 \mathrm{~mL} / \mathrm{kg}$ de peso predicho, con $6-8 \mathrm{cmH}_{2} \mathrm{O}$ de PEEP). El resultado fue menos complicaciones (10.5\%) pulmonares y extrapulmonares con Vt de 6-8 $\mathrm{mL} / \mathrm{kg}$ de peso predicho respecto al grupo de Vt de $10-12 \mathrm{~mL} / \mathrm{kg}$ de peso predicho $(27.5 \%)$. Estos resultados están respaldados por un reciente metaanálisis que documenta disminución de las complicaciones pulmonares postoperatorias RR 0.64 (IC95\% 0.46 a 0.88; $p=0.01$ ) en los pacientes que utilizan Vt bajo ( $\leq 8 \mathrm{~mL} / \mathrm{kg}$ peso predicho). Aunque al parecer el nivel de PEEP alto $\left(\geq 5 \mathrm{cmH}_{2} \mathrm{O}\right)$ versus PEEP bajo $\left(\leq 5 \mathrm{cmH}_{2} \mathrm{O}\right)$ no influye en los resultados RR 0.93 (IC95\% 0.64 a 1.37; $p=0.72$ ). No obstante, necesitamos más estudios para poder determinar el nivel de PEEP adecuado durante el intraoperatorio para prevenir complicaciones postoperatorias. ${ }^{39}$

\section{CONCLUSIONES}

El uso de volumen corriente intermedio ( $>8$ a índices de oxigenación: $\mathrm{PaO}_{2} / \mathrm{FiO}_{2}$ y $\mathrm{PaO}_{2} / \mathrm{PAO}_{2}$ podría ser el comienzo del VILI. La alteración de los índices de oxigenación está correlacionada con la estrategia ventilatoria (Vtl y VtB), pero no al nivel de PEEP o balance de líquidos. No existe diferencia entre el grupo de Vtl y el grupo de VtB con respecto al tiempo de ventilación mecánica, estancia en la unidad de cuidados intensivos o mortalidad. El nivel de PEEP durante el perioperatorio de los pacientes neuroquirúrgicos no ocasiona diferencia significativa en la escala de coma de Glasgow.

\section{BIBLIOGRAFÍA}

1. Slutsky AS. History of mechanical ventilation: from vesalius to ventilator-induced lung injury. Am J Respir Crit Care Med. 2015;191(10):1106-1115.

2. Soto G. Ventilación mecánica: una breve historia. Neumol Pediatr. 2016;11(4):151-154.

3. Marini JJ. Mechanical ventilation: past lessons and the near future. Crit Care. 2013;17(Suppl 1):S1.

4. Thille WA, Rodriguez P, Cabello B, Lellouche F, Brochard L. Patient-ventilator asynchrony during assisted mechanical ventilation. Intensive Care Med. 2006;32(10):1515-1522.

5. Pettenuzzo T, Fan E. Year in review: mechanical ventilation. Respir Care. 2017;62(5):629-635.

6. Nieman GF, Gatto LA, Habashi NM. Impact of mechanical ventilation on the pathophysiology of progressive acute lung injury. J Appl Physiol. 2015;119(11):1245-1261.

7. Kuchnicka K, Maciejewski D. Ventilator-associated lung injury. Anaesthesiol Intensive Ther. 2013;45(3):164-170.

8. Serpa Neto A, Hemmes SN, Barbas CS, Beiderlinden M, Fernandez-Bustamante A, Futier E, et al. Incidence of mortality and morbidity related to postoperative lung injury in patients who have undergone abdominal or thoracic surgery: a systematic review and meta-analysis. Lancet Respir Med. 2014;2(12):10071015.

9. Hemmes SN, Serpa Neto A, Schultz MJ. Intraoperative ventilatory strategies to prevent postperative pulmonary cmplications: a meta-analysis. Curr Opin Anesthesiol. 2013;26:126-133.

10. Gajic O, Frutos-Vivar F, Esteban A, Hubmayr RD, Anzueto A. Ventilator settings as a risk factor for acute respiratory distress 
syndrome in mechanically ventilated patients. Intensive Care Med. 2005;31(7):922-926.

11. Protti A, Votta E, Gattinoni L. Which is the most important strain in the pathogenesis of ventilator-induced lung injury: dynamic or static? Curr Opin Crit Care. 2014;20(1):33-38.

12. Slutsky AS, Ranieri VM. Ventilator-induced lung injury. N Engl J Med. 2013;369:2126-2136.

13. Baid $\mathrm{H}$. Patient safety. Identifying and managing complications of mechanical ventilation. Crit Care Nurs Clin North Am. 2016;28(4):451-462.

14. Neto AS, Hemmes SN, Barbas CS, Beiderlinden M, FernandezBustamante A, Futier E, et al. Association between driving pressure and development of postoperative pulmonary complications in patients undergoing mechanical ventilation for general anaesthesia: a meta-analysis of individual patient data. Lancet Respir Med. 2016;4(4):272-280.

15. Rao VK, Khanna AK. Postoperative respiratory impairment is a real risk for our patients: the intensivist's perspective. Anesthesiol Res Pract. 2018:3215923.

16. Fernandez-Bustamante A, Wood CL, Tran ZV, Moine P. Intraoperative ventilation: incidence and risk factors for receiving large tidal volumes during general anesthesia. BMC Anesthesiol. 2011;11:22

17. Hahn CE. KISS and indices of pulmonary oxygen transfer. $\mathrm{Br} J$ Anaesth. 2001;86:465-467.

18. Manzo PE, Mayo MR, de la Cruz LJ. La corrección del índice de oxigenación en los pacientes críticos al nivel de la ciudad de México. Rev Asoc Mex Med Crit y Ter Int. 2008;22(1):26-35.

19. Colín EV, Monares ZE, Sánchez CC, Elizalde GJJ, Poblano MM, Aguirre SJ, et al. Índices de oxigenación como factores pronósticos en el síndrome de insuficiencia respiratoria aguda. Rev Asoc Mex Med Crit y Ter Int. 2008;22(3):131-142.

20. Boone MD, Jinadasa SP, Mueller A, Shaefi S, Kasper EM, Hanafy KA, et al. The effect of positive end-expiratory pressure on intracranial pressure and cerebral hemodynamics. Neurocrit Care. 2017;26(2):174-181.

21. Peris-Montalt R, Cruz-García-Dihinx I, Errando C, Granell M. Efectos de la ventilación mecánica intraoperatoria y de la ventilación de protección pulmonar en el paciente quirúrgico adulto. MÉD. UIS. 2015;28(1):65-78.

22. Ranieri VM, Suter PM, Tortorella C, De Tullio R, Dayer JM, Brienza A, et al. Effect of mechanical ventilation on inflammatory mediators in patients with acute respiratory distress syndrome: a randomized controlled trial. JAMA. 1999;282:54-61.

23. The Acute Respiratory Distress Syndrome Network, Brower RG, Matthay MA, Morris A, Schoenfeld D, Thompson BT. A. Ventilation with lower tidal volumes as compared with traditional tidal volumes for acute lung injury and the acute respiratory distress syndrome. N Engl J Med. 2000;342:1301-1308.

24. Simonis FD, Serpa Neto A, Binnekade JM, Braber A, Bruin $\mathrm{KCM}$, Determann RM, et al. Effect of a low vs intermediate tidal volume strategy on ventilator-free days in Intensive Care Unit patients without ARDS: a randomized clinical trial. JAMA. 2018;320(18):1872-1880.

25. Fernandez-Perez ER, Keegan MT, Brown DR, Hubmayr RD, Gajic O. Intraoperative tidal volume as a risk factor for respiratory failure after pneumonectomy. Anesthesiology. 2006;105:14-18.

26. Michelet P, D' Journo XB, Roch A, Doddoli C, Marin V, Papazian $\mathrm{L}$, et al. Protective ventilation influences systemic inflammation after esophagectomy: a randomized controlled study. Anesthesiology. 2006;105:911-919.

27. M Karcz, PJ Papadakos. Respiratory complications in the postanesthesia care unit: A review of pathophysiological mechanisms. Can J Respir Ther. 2013;49(4):21-29.
28. Armstrong J, Guleria A, Girling K. Evaluation of gas exchange deficit in the critically ill. Continuing Education in Anaesthesia Critical Care \& Pain. 2007;7:131-134.

29. Artigas A, Bernard GR, Carlet J, Carlet J, Falke K, Hudson L, et al. The American-European Consensus Conference on ARDS. Definitions, mechanisms, relevant outcomes, and clinical trial coordination. Am J Respir Crit Care Med. 1994;157:1332-1347.

30. Schmidt GA, Girard TD, Kress JP, Morris PE, Ouellette DR, Alhazzani W, et al. Liberation From Mechanical Ventilation in Critically III Adults: Executive Summary of an Official American College of Chest Physicians/American Thoracic Society Clinical Practice Guideline. Chest. 2017;151(1):160-165.

31. Cortés-Román JC, Sánchez-Díaz JS, Castañeda-Valladares E, Peniche-Moguel KG, Gutiérrez-Jiménez AA, Calyeca-Sánchez $M V$. Índices de oxigenación como predictores de fracaso en la extubación en pacientes críticamente enfermos. Acta Colombiana de Cuidado Intensivo. 2018;18(3):131-198.

32. Sánchez CM, Quintana DM, Palacios D, Hortigüela V, Schulke $\mathrm{CM}$, García J, et al. Relationship between the alveolar-arterial oxygen gradient and $\mathrm{PaO}_{2} / \mathrm{FiO}_{2}$-introducing PEEP into the model. Med Intensiva. 2012;36(5):329-334.

33. Sganga G, Siegel JH, Coleman B, Giovannini I, Boldrini G, Pittiruti M. The physiologic meaning of the respiratory index in various types of critical illness. Crit Shock. 1985;17:179-193.

34. Cane RD, Shapiro BA, Templin R, Walther K. Unreliability of oxygen tension-based indices in reflecting intrapulmonary shunting in critically ill patients. Crit Care Med. 1988;16:12431245.

35. Rodríguez-Botoa G, Rivero-Garvía M, Gutiérrez-González R, Márquez-Rivas J. Conceptos básicos sobre la fisiopatología cerebral y la monitorización de la presión intracraneal. Neurología. 2015;30(1):16-22.

36. Slack RS, Shucart W. Respiratory dysfunction associated with traumatic injury to the central nervous system. Clin Chest Med. 1994;15:739-749.

37. Corradi F, Robba C, Tavazzi G, Via G. Combined lung and brain ultrasonography for an individualized "brain-protective ventilation strategy" in neurocritical care patients with challenging ventilation needs. Crit Ultrasound J. 2018;10(1):24.

38. Futier E, Constantin J, Paugam-Burtz C, Pascal J, Eurin M, Neuschwander A, et al. A trial of intraoperative low-tidal-volume ventilation in abdominal surgery. N Engl J Med. 2013;369:428437.

39. Serpa Neto A, Hemmes SN, Barbas CS, Beiderlinden M, Biehl M, Binnekade JM, et al. Protective versus conventional ventilation for surgery: a systematic review and individual patient data metaanalysis. Anesthesiology. 2015;123(1):66-78.

Patrocinios. Relación de conflicto de intereses: El presente trabajo no recibió patrocinios, sólo se utilizaron recursos propios de nuestro hospital. Los autores declaramos no tener conflicto de intereses.

Correspondencia:

Jesús Salvador Sánchez Díaz

Av. Cuauhtémoc SN,

Formando Hogar, 91897, Veracruz, Ver.

Tel: 2299342031

E-mail: drsalvadorsanchezdiaz@gmail.com 